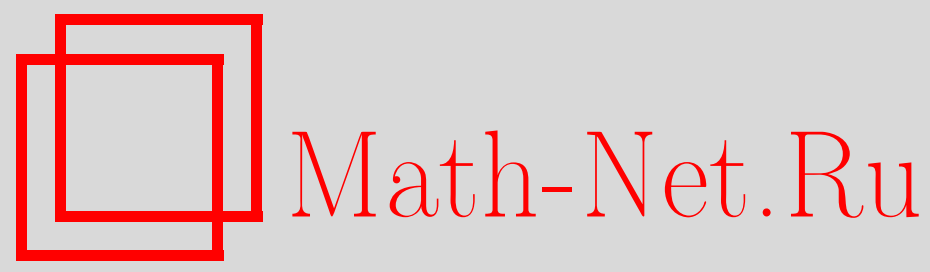

В. И. Парусников, Многогранники Клейна для четвертой экстремальной кубической формы, Матем. заметки, 2000, том 67, выпуск 1, 110-128

DOI: https://doi.org/10.4213/mzm819

Использование Общероссийского математического портала Math-Net.Ru подразумевает, что вы прочитали и согласны с пользовательским соглашением http://www.mathnet.ru/rus/agreement

Параметры загрузки:

IP : 18.234 .197 .8

26 апреля 2023 г., 13:39:00

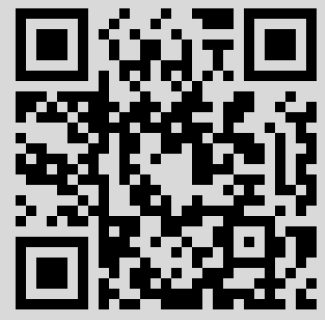




\title{
МНОГОГРАННИКИ КЛЕЙНА ДЛЯ ЧЕТВЕРТОЙ ЭКСТРЕМАЛЬНОЙ КУБИЧЕСКОЙ ФОРМЫ
}

\section{В.И. Парусников}

\begin{abstract}
Давенпортом и Свиннертоном-Дайером были найдены первые 19 экстремальных тернарных кубических форм $g_{i}$, имеющих тот же смысл, что и известные формы Марковав бинарном квадратичном случае. Многогранники Клейна для форм $g_{1}, g_{2}, g_{3}$ были недавно вычислены Брюно и Парусниковым. Они же для кратных векторов вычислили "неполные частные", полученные по разным матричным обобщениям алгоритма цепных дробей, и изучили их расположение относительно многогранников Клейна. Здесь рассматриваются аналогичные задачи для четвертой формы $g_{4}$. А именно, вычислены многогранники Клейна для $g_{4}$ и сопряженной ей формы $g_{4}^{*}$. Они оказались существенно разными. Найдены их периоды и фундаментальные области. Вычислены разложения векторов этих форм по матричным алгоритмам.
\end{abstract}

Библиография: 20 названий.

1. Введение. Наилучшие рациональные приближения $p / q$ к числу $\alpha$ суть подходящие дроби $p_{k} / q_{k}$ цепной дроби числа $\alpha$ [1]. Клейн [2]-[4] предложил следующую геометрическую интерпретацию цепных дробей (см. также [5, гл. III, п. 19]). Пусть на плоскости координат $p, q$ проведены прямые $\mathcal{L}_{1}=\{p, q: p=\alpha q\}$ и $\mathcal{L}_{2}=\{p, q: q=0\}$. Они образуют смежные углы $\mathscr{O}_{1}=\{p, q: p \geqslant \alpha q, q \geqslant 0\}$ и $\mathscr{O}_{2}=\{p, q: p \leqslant \alpha q, q \geqslant 0\}$. Обозначим $K_{i}$ выпуклую оболочку целочисленных точек (без точки $\left.(0,0)\right)(p, q)$, попавших в $\mathscr{O}_{i}$. Границы $\partial K_{i}$ множеств $K_{i}$ суть выпуклые ломанные линии. Вершинам $(p, q)$ этих ломанньх отвечают подходящие дроби $p / q$ цепной дроби числа $\alpha$. Число целочисленных точек на ребре минус один равно неполному частному цепной дроби и т.д. Там же Клейн [2], [3] предложил трехмерньй аналог этой конструкции для изучения наилучших приближений. А именно, пусть в $\mathbb{R}^{3}$ заданы три пересекаюшиеся в нуле плоскости $\mathcal{L}_{i}=\left\{X:\left\langle L_{i}, X\right\rangle=0, i=1,2,3\right\}$. Здесь $X, L \in \mathbb{R}^{3}$ и $\langle.,$.$\rangle означает скалярное про-$ изведение. Каждому набору $\Sigma=\left(\sigma_{1}, \sigma_{2}, \sigma_{3}\right)$, где $\sigma_{i}= \pm 1$, соответствует свой октант $\mathscr{O}_{\Sigma}=\left\{X: \sigma_{i}\left\langle L_{i}, X\right\rangle \geqslant 0, i=1,2,3\right\}$, ограниченный этими плоскостями. В каждом октанте $\mathscr{O}_{\Sigma}$ рассматривается выпуклая оболочка $K_{\Sigma}$ всех целочисленных точек $X \in \mathscr{O}_{\Sigma}$, $X \neq 0$. Ее граница $\partial K_{\Sigma}$ является вьпуклой двумерной многогранной поверхностью, состоящей из вершин, ребер и граней. Ее вершины должны давать наилучшие рациональные приближения для линейных форм $\left\langle L_{i}, X\right\rangle$.

Рассмотрим теперь кубическую однородную форму $g(X)$, являющуюся произведением трех вешественных линейных форм: $g(X)=\left\langle L_{1}, X\right\rangle\left\langle L_{2}, X\right\rangle\left\langle L_{3}, X\right\rangle$. Пусть $\Delta=$

Работа выполнена при финансовой поддержке Российского фонда фундаментальных исследований, гранты № 97-01-00268, № 99-01-01063. 
$\operatorname{det}\left(L_{1} L_{2} L_{3}\right)$ и $\mu(g)$ - наименьшее значение величины $\left|g \Delta^{-1}\right|$ на целочисленных $X, X \neq$ 0 :

$$
\mu(g)=\frac{1}{|\Delta|} \min _{X \in \mathbb{Z}^{n} \backslash 0}|g(X)| .
$$

Далее векторы записываются как матрицы-строки, но в матричных операциях рассматриваются как матрицы-столбцы. Давенпорт [6]-[8] доказал, что $\mu(g) \leqslant 1 / 7$ и $\mu(g)=1 / 7$ только для формы $g_{1}$, у которой

$$
L_{i}=\left(1, \lambda_{i}, \lambda_{i}^{2}\right), \quad i=1,2,3,
$$

где $\lambda_{i}-$ суть корни уравнения $\lambda^{3}+\lambda^{2}-2 \lambda-1=0$, и для эквивалентных форм. Если их исключить из рассмотрения, то $\mu(g) \leqslant 1 / 9$ и $\mu(g)=1 / 9$ только для формы $g_{2}, \mathrm{y}$ которой $L_{i}$ имеют вид $(2)$, но $\lambda_{i}$ суть корни уравнения $\lambda^{3}-3 \lambda-1=0$, и для эквивалентных форм. Свиннертон-Дайер [9] численно отыскал первые 19 форм $g_{i}$ с наибольшими значениями $\mu$.

В работах [10]-[14] для форм $g_{1}, g_{2}$ и $g_{3}$ были вычислены многогранники Клейна и разложения векторов $L_{3}$ по матричным алгоритмам Эйлера, Якоби, Пуанкаре, Бруна, Брюно и Парусникова. Изучалось расположение их подходящих дробей относительно многогранников Клейна. В [15] аналогичная работа была сделана для формы $h_{4}$, порожденной тем же полем, что и четвертая экстремальная форма $g_{4}$. В настоящей работе вычисляются многогранники Клейна формы $g_{4}$, ее сопряженной формы $g_{4}^{*}$ и разложения их векторов по матричным алгоритмам. При этом впервые встречается ситуация, когда многогранники Клейна в сопряженных пространствах $\mathbb{R}^{n}$ и $\mathbb{R}_{*}^{n}$ существенно различны.

Предварительной публикацией настоящей работы является [16].

2. Многогранники Клейна. О выпуклых многогранниках и конусах см. [17], [18]. Напомним определения, данные в [14]. Рассмотрим два $n$-мерных вещественных пространства $\mathbb{R}^{n}$ и $\mathbb{R}_{*}^{n}$ с координатами $X$ и $U$ соответственно и со скалярным произведением $\langle U, X\rangle=u_{1} x_{1}+\cdots+u_{n} x_{n}$ для $U=\left(u_{1}, \ldots, u_{n}\right) \in \mathbb{R}_{*}^{n}$ и $X=\left(x_{1}, \ldots, x_{n}\right) \in \mathbb{R}^{n}$. Пусть в $\mathbb{R}_{*}^{n}$ заданы $n$ линейно независимых векторов $L_{1}, \ldots, L_{n}$. Каждому из $2^{n}$ наборов $\Sigma=\left(\sigma_{1}, \ldots, \sigma_{n}\right)$, где $\sigma_{i}= \pm 1$, соответствует в $\mathbb{R}_{*}^{n}$ свой вьпукльй конус

$$
\mathscr{O}_{\Sigma}^{*}=\left\{U: U=\alpha_{1} \sigma_{1} L_{1}+\cdots+\alpha_{n} \sigma_{n} L_{n}, \alpha_{i} \geqslant 0\right\}
$$

В случаях конкретных $\sigma_{i}$ вместо чисел +1 и -1 мы будем писать “+" и “-". Отвечающие векторам $L_{i}$ плоскости $\mathcal{L}_{i}=\left\{X:\left\langle L_{i}, X\right\rangle=0\right\}, i=1, \ldots, n$, делят пространство $\mathbb{R}^{n}$ на $2^{n}$ выпуклых конусов

$$
\mathscr{O}_{\Sigma}=\left\{X:\left\langle\sigma_{i} L_{i}, X\right\rangle \geqslant 0, i=1, \ldots, n\right\}
$$

Пусть векторы $M_{1}, \ldots, M_{n}$ ортогональны векторам $L_{1}, \ldots, L_{n}$, т.е.

$$
\left\langle L_{i}, M_{j}\right\rangle=\delta_{i j} \psi_{i}, \quad i, j=1, \ldots, n,
$$

где константы $\psi_{i}$ не равны нулю, а $\delta_{i j}$ - символ Кронекера. Тогда конусы $\mathscr{O}_{\Sigma}^{*}$ и $\mathscr{O}_{\Sigma}$ можно задавать как

$$
\begin{aligned}
& \mathscr{O}_{\Sigma}^{*}=\left\{U:\left\langle U, M_{i} \sigma_{i} \psi_{i}\right\rangle \geqslant 0, i=1, \ldots, n\right\}, \\
& \mathscr{O}_{\Sigma}=\left\{X: X=\alpha_{1} \psi_{1} \sigma_{1} M_{1}+\cdots+\alpha_{n} \psi_{n} \sigma_{n} M_{n}, \alpha_{i} \geqslant 0\right\} .
\end{aligned}
$$


Рассмотрим вьпуклую оболочку $K_{\Sigma}$ всех попадающих в конус $\mathscr{O}_{\Sigma}$ целочисленных точек $X \in \mathbb{Z}^{n}, X \neq 0$. Многогранником Клейна назовем $(n-1)$-мерную многогранную поверхность $\partial K_{\Sigma}$, являющуюся границей множества $K_{\Sigma}$. В двойственном конусе $\mathscr{O}_{\Sigma}^{*}$ выпуклую оболочку целочисленных точек $U \in \mathbb{Z}_{*}^{n}, U \neq 0$, обозначим через $K_{\Sigma}^{*}$. Кроме того в $\mathscr{O}_{\Sigma}^{*}$ и $\mathscr{O}_{\Sigma}$ определим многогранники

$$
\begin{aligned}
& \bar{K}_{\Sigma}^{*}=\left\{U: U \in \mathbb{R}^{n},\langle U, X\rangle \geqslant 1 \text { для всех } X \in K_{\Sigma}\right\}, \\
& \bar{K}_{\Sigma}=\left\{X: X \in \mathbb{R}^{n},\langle U, X\rangle \geqslant 1 \text { для всех } U \in K_{\Sigma}^{*}\right\},
\end{aligned}
$$

двойственные многогранникам $K_{\Sigma}$ и $K_{\Sigma}^{*}$. Вершины последних могут быть нецелыми точками. Так как $\langle U, X\rangle \geqslant 0$ для $X \in \mathscr{O}_{\Sigma}$ и $U \in \mathscr{O}_{\Sigma}^{*}$, а для $X \in Z^{n}, U \in Z_{*}^{n}\langle U, X\rangle-$ целое, то $\bar{K}_{\Sigma}^{*} \supset K_{\Sigma}^{*}$ и $\bar{K}_{\Sigma} \supset K_{\Sigma}$.

Поверхность $\partial K_{\Sigma}$ состоит из граней $\Gamma_{j}^{(d)}$ разных размерностей $d, 0 \leqslant d<n-1$ : вершин $(d=0)$, ребер $(d=1), \ldots$, гиперграней $(n-1)$.

Целочисленная точка называется точкой относительного минимума, если в этом же октанте нет целочисленных точек, лежаших ближе, чем она, одновременно ко всем ограничивающим октант гиперплоскостям $\mathcal{L}_{i}$.

Пусть целочисленные точки $X_{1}, \ldots, X_{n}$ лежат в гиперплоскости $\mathcal{L}$, служащей продолжением грани $\Gamma_{j}^{(n-1)}$, а векторы $X_{i}-X_{1}, i=2, \ldots, n$, образуют базис этой гиперплоскости, т.е. $\mathrm{y}(n-1) \times n$ матрищы со строками из этих векторов наибольший общий делитель всех миноров размера $n-1$ равен единице. Величину $\rho\left(\Gamma_{j}^{(n-1)}\right)=$ $\left|\operatorname{det}\left(X_{1} X_{2} \ldots X_{n}\right)\right|$ назовем расстоянием гиперграни $\Gamma_{j}^{(n-1)}$.

Отметим следующее соответствие: каждой грани $\Gamma_{j}^{(n-d-1)}$ многогранника $\bar{K}_{\Sigma}^{*}$ отвечает некоторая грань $\Gamma_{k}^{(d)}$ многогранника $K_{\Sigma}$, причем $\langle U, X\rangle=1$ для всех $U \in \Gamma_{j}^{(n-d-1) *}$ и $X \in \Gamma_{k}^{(d)}$. Заметим еще, что каждая грань является вьпуклой оболочкой своих вершин.

Пересечения $\partial K_{\Sigma} \cap \partial \bar{K}_{\Sigma}$ и $\partial K_{\Sigma}^{*} \cap \partial \bar{K}_{\Sigma}^{*}$ содержат все гиперграни с расстоянием $\rho=1$. Алгоритм вычисления таких пересечений гиперграней состоит в следующем. Далее набор $\Sigma$ фиксируем и индекс $\Sigma$ опускаем. Пусть точка $X_{1} \in \partial K \cap \partial \bar{K}$; находим все целочисленные решения $\widetilde{U}_{1}, \ldots, \widetilde{U}_{m} \in \mathscr{O}^{*} \cap \mathbb{Z}_{*}^{n}$ уравнения $\left\langle U, X_{1}\right\rangle=1$ и $n$ неравенств из (6): их вьпуклая оболочка есть некотораягрань $\Gamma_{j}^{(d) *}$ поверхности $\partial K^{*}$. Среди точек $\widetilde{U}_{1}, \ldots, \widetilde{U}_{m}$ выделим все вершины $U_{1}, \ldots, U_{r}$ грани $\Gamma_{j}^{(d) *}$. Для каждой из них составляем свое уравнение

$$
\left\langle U_{i}, X\right\rangle=1
$$

и находим все его решения $X \in \mathscr{O} \cap \mathbb{Z}^{n}$, т.е. целочисленные решения $X$ уравнения (7), удовлетворяющие $n$ неравенствам из (4). Получаем точки $\widetilde{X}_{1}, \ldots, \widetilde{X}_{k}$, лежашие на одной грани. Выбираем из них вершины $X_{2}, \ldots, X_{s}$. Для каждой из них ищем все решения $U \in \mathscr{O} \cap \mathbb{Z}_{*}^{n}$ уравнения $\left\langle U, X_{i}\right\rangle=1$ и т.д. Во многих случаях так удается вычислить все целочисленные точки многогранника Клейна $\partial K$. Весь многогранник $\partial K$ однозначно восстанавливается как их выпуклая оболочка.

3. Формы и модули. Напомним некоторые определения из теории чисел [19], [20]. Пусть $\mathbb{Z}[x]$ - кольцо многочленов от $x$ с цельми коэффициентами. Корни многочленов из $\mathbb{Z}[x]$ назьваются алгебраическими числами и, если старший коэффициент многочлена равен 1, иельми алгебраическими числами. 
Степень $n$ неприводимого над $\mathbb{Z}[x]$ многочлена $p(x)$, корнем которого является некоторое число $\lambda$, называется степенью числа $\lambda$; другие $n-1$ корней $\lambda^{(j)}$ многочлена $p$ будут алгебраическими числами, сопряженным. числу $\lambda=\lambda^{(1)}$ (в поле рачиональных чисел $\mathbb{Q})$.

Линейные комбинации степеней $\sum_{j=0}^{n-1} a_{k} \lambda^{k}, a_{k} \in \mathbb{Q}$, образуют поле - расширение $\mathbb{Q}(\lambda)$ поля $\mathbb{Q} ;$ число $n$ называется степенью поля, а поля $\mathbb{Q}\left(\lambda^{(j)}\right)$ - полями, сопряженны.ми к $\mathbb{Q}(\lambda)$. Фиксированная нумерация корней многочлена $p$ задает нумерацию полей. Набор элементов поля, через который с рациональными коэффициентами выражаются все элементы поля, назьвается базисом поля. Заданное на базисе $1, \lambda^{(j)}, \ldots,\left(\lambda^{(j)}\right)^{n-1}$ отображение $\left(\lambda^{(1)}\right)^{k} \rightarrow\left(\lambda^{(j)}\right)^{k}, k=0, \ldots, n-1, j=1, \ldots, n$, определяет естественный изоморфизм полей, при этом числу поля $\alpha^{(1)}$ соответствует $\alpha^{(k)}$ - conряжсенное $\kappa$ нему в поле $\mathbb{Q}(\lambda)$. Норма $\operatorname{Norm}(\alpha)$ алгебраического числа $\alpha$ это рациональное число - произведение $\alpha$ и всех его сопряженных чисел:

$$
\operatorname{Norm}(\alpha)=\alpha^{(1)} \cdots \alpha^{(n)} .
$$

Если все корни многочлена $p$ вещественные, поле $\mathbb{Q}(\lambda)$ называется вполне вещественным. Отображение, сопоставляющее элементу $\alpha$ поля $n$-мерньй вектор $\left(\alpha^{(1)}, \ldots\right.$, $\left.\alpha^{(n)}\right) \in \mathbb{R}^{n}$, назьвается каноническим вложением поля $\mathbb{Q}(\lambda)$.

Пусть даны $v_{1}, \ldots, v_{m} \in \mathbb{Q}(\lambda)$ или, что то же самое, задан вектор $V=\left(v_{1}, \ldots, v_{m}\right) \in$ $\mathbb{Q}^{m}(\lambda)$. Модулем (точнее, $\mathbb{Z}$-модулем) $N$ с образующ, ми $v_{j}$ в поле $\mathbb{Q}(\lambda)$ назьвается множество целочисленньх линейных комбинаций

$$
N=\left\{\sum_{j=1}^{m} y_{j} v_{j}, Y=\left(y_{1}, \ldots, y_{m}\right) \in \mathbb{Z}^{m}\right\} .
$$

Говорят, что модули подобны, если у них имеются пропорциональные системы образующих. Векторы $V^{(j)}=\left(v_{1}^{(j)}, \ldots, v_{m}^{(j)}\right) \in \mathbb{Q}^{m}\left(\lambda^{(j)}\right)$ с компонентами из сопряженных полей задают модули, сопряженные с $N$. Точки модуля интерпретируются как значения линейной формы $\langle V, Y\rangle$ на всевозможных целочисленных векторах $Y$. Модуль полный, если максимальное число его линейно независимых образующих $m$ равно степени $n$ поля.

Каждому модулю $N$ вполне вещественного поля однозначно соответствуют два объекта. Первьй объект это $m$-мерная решетка в $\mathbb{R}^{n}-$ множество проекций точек модуля при каноническом вложении

$$
\left\{\left(\sum_{j=1}^{m} y_{j} v_{j}^{(1)}, \ldots, \sum_{j=1}^{m} y_{j} v_{j}^{(n)}\right) \in \mathbb{R}^{n}, y_{j} \in \mathbb{Z}\right\} .
$$

Второй объект - разложимая форма. Однородная функция (форма) степени $k \in \mathbb{N}$ от $n$ переменных назьвается разложимой формой, если она раскладывается в произведение линейных форм с коэффициентами - алгебраическими числами из некоторого расширения $\mathbb{Q}(\beta)$ поля $\mathbb{Q}$. Форма назьвается иелочисленной, если ее значения на множестве $\mathbb{Z}^{n}$ - целые числа. Модулю $N$ соответствует разложимая форма степени $n$

$$
g_{N}(Y)=\operatorname{Norm}\left(\sum_{j=1}^{m} y_{j} v_{j}\right)=\operatorname{Norm}(\langle V, Y\rangle) .
$$


Если образующие модуля $N$ - целые алгебраические числа, значения формы $g_{N}(Y)$ на векторах из $\mathbb{Z}^{n}$ - целые числа. Верно и обратное, каждая полная разложимая форма с точностью до множителя - рационального числа может быть интерпретирована как норма элементов некоторого модуля с образующими - цельми числами из некоторого расширения поля $\mathbb{Q}$. Подобным модулям отвечают подобные решетки и пропорциональные (с коэффициентом - рациональным числом) разложимые формы. Значения коэффициентов $\mu(g)$ для подобных форм одно и то же.

Заметим, что поле $\mathbb{Q}(\beta)$, где возможно разложение формы на линейные множители, это минимальное поле алгебраических чисел, содержащее как образующие модуля, так и числа, сопряженные к ним.

4. Форма $g_{4}(X)$. Экстремальные формы $g_{i}$ Свиннертона-Дайера [9] задаются трехмерными решетками. Образующими модулей, отвечающих решеткам, служат 1 и числа $\alpha, \beta$ из некоторого кубического поля $\mathbb{Q}(\lambda)$. Для каждой из форм $g_{i}$ числа $\lambda, \alpha, \beta$ свои.

Для четвертой формы число $\lambda$ удовлетворяет кубическому уравнению

$$
f(\lambda) \stackrel{\text { def }}{=} \lambda^{3}+9 \lambda^{2}+6 \lambda-1=0 .
$$

Дискриминант поля $\mathbb{Q}(\lambda)$ положителен (равен $\left.63^{2}=3969\right)$, все корни уравнения $(8)$ вешественны и поле $\mathbb{Q}(\lambda)$ - вполне вещественное. Выписанные в порядке возрастания корни $\lambda_{j}=\lambda^{(j)}$ уравнения (8) суть

$$
\left(\lambda_{1}, \lambda_{2}, \lambda_{3}\right) \approx(-8.2588452612,-0.87891769753,0.13776295871) .
$$

Так как $\lambda_{j}=-1 /\left(1+\lambda_{j+1}\right)=\lambda_{j+1}^{2}+8 \lambda_{j+1}-2, j=1,2,3, \lambda_{0}=\lambda_{3}$, числа $\lambda_{j}$ лежат в одном и том же кубическом поле. Это значит, что $\mathbb{Q}\left(\lambda^{(j)}\right)=\mathbb{Q}\left(\lambda^{(1)}\right)$, т.е. рассматриваемое поле $\mathbb{Q}(\lambda)$ нормально. Образующими модуля берутся числа

$$
1, \quad \alpha=\frac{-3+15 \lambda+2 \lambda^{2}}{5}, \quad \beta=\frac{11+10 \lambda+\lambda^{2}}{5} .
$$

Числа $\alpha, \beta$ - не целые алгебраические числа: $\alpha^{3}+1.2 \alpha^{2}-5.4 \alpha-1=0, \beta^{3}-2.4 \beta^{2}-$ $0.6 \beta+1=0$. Для $\lambda=\lambda_{j}, j=1,2,3$, они численно равны

$$
\begin{aligned}
\left(\alpha_{1}, \alpha_{2}, \alpha_{3}\right) & \approx(1.90687423572,-2.92775456497,-0.17911967075), \\
\left(\beta_{1}, \beta_{2}, \beta_{3}\right) & \approx(-0.67598551273,0.59666386875,2.47932164398) .
\end{aligned}
$$

Приведем также численные значения векторов, задающих три сопряженных модуля:

$$
L_{j}=\left(1, \alpha_{j}, \beta_{j}\right), \quad j=1,2,3,
$$

т.e.

$$
\begin{aligned}
& L_{1} \approx(1,1.90687423572,-0.67598551273), \\
& L_{2} \approx(1,-2.92775456497,0.59666386875), \\
& L_{3} \approx(1,-0.17911967075,2.47932164398) .
\end{aligned}
$$

В [20] была найдена симметричная матрица $A$ с характеристическим уравнением (8):

$$
A=\left(\begin{array}{ccc}
-1 & 0 & 1 \\
0 & 0 & 1 \\
1 & 1 & -8
\end{array}\right)
$$


Ее собственные векторы - это ортогональные (в силу симметричности $A$ ) векторы

$$
L_{j}^{\prime \prime}=\left(1,7+9 \lambda_{j}+\lambda_{j}^{2}, 1+\lambda_{j}\right), \quad j=1,2,3,
$$

т.е.

$$
\begin{aligned}
& L_{1}^{\prime \prime} \approx(1,0.878917698,-7.258845261), \\
& L_{2}^{\prime \prime} \approx(1,0.137762959,0.121082302), \\
& L_{3}^{\prime \prime} \approx(1,8.258845261,1.137762959) .
\end{aligned}
$$

Чтобы избежать дополнительных буквенных обозначений, договоримся что обозначающая матрицу буква, заключенная в круглые скобки и снабженная парой индексов, означает элемент матрицы, и снабженная одним индексом - столбец матрицы. Введем матрицы

$$
B_{1}=\frac{1}{5}\left(\begin{array}{ccc}
5 & 0 & 0 \\
-3 & 15 & 2 \\
11 & 10 & 1
\end{array}\right), \quad B_{2}=\left(\begin{array}{lll}
1 & 0 & 0 \\
7 & 9 & 1 \\
1 & 1 & 0
\end{array}\right),
$$

$L^{\prime \prime}, L:\left(L^{\prime \prime}\right)_{i j}=\left(L_{j}^{\prime \prime}\right)_{i},(L)_{i j}=\left(L_{j}\right)_{i}$, а также матрицу Вандермонда $W$ и диагональную матрицу $\Lambda$

$$
W=\left(\begin{array}{ccc}
1 & 1 & 1 \\
\lambda_{1} & \lambda_{2} & \lambda_{3} \\
\lambda_{1}^{2} & \lambda_{2}^{2} & \lambda_{3}^{2}
\end{array}\right), \quad \Lambda=\left(\begin{array}{ccc}
\lambda_{1} & 0 & 0 \\
0 & \lambda_{2} & 0 \\
0 & 0 & \lambda_{3}
\end{array}\right)
$$

Тогда

$$
A L^{\prime \prime}=L^{\prime \prime} \Lambda, \quad L^{\prime \prime *} A=\Lambda L^{\prime \prime *}
$$

(звездочка $*$ означает транспонирование), а соотношения $(9),(10)$ и (12) записьваются в виде $L=B_{1} W, L^{\prime \prime}=B_{2} W$. Коэффициенты матрицы $B_{1}$ - рациональные, а матрищ $B_{1}^{-1} 4, B_{2}, B_{2}^{-1}$ - целые числа:

$$
B_{1}^{-1}=\left(\begin{array}{ccc}
1 & 0 & 0 \\
-5 & -1 & 2 \\
39 & 10 & -15
\end{array}\right), \quad B_{2}^{-1}=\left(\begin{array}{ccc}
1 & 0 & 0 \\
-1 & 0 & 1 \\
2 & 1 & -9
\end{array}\right) .
$$

Из этих соотношений можно найти матрицу $A_{1}$, для которой собственньми векторами (правыми) будут векторы $L_{j}$, т.е. $A_{1} L=L \Lambda$. Поскольку $W=B_{1}^{-1} L=B_{2}^{-1} L^{\prime \prime}$, то $L^{\prime \prime}=B_{2} B_{1}^{-1} L$. Следовательно, по (13) $A B_{2} B_{1}^{-1} L=B_{2} B_{1}^{-1} L \Lambda$, т.е.

$$
A_{1}=B_{1} B_{2}^{-1} A B_{2} B_{1}^{-1}=\left(\begin{array}{ccc}
-5 & -1 & 2 \\
-8 & -3 & 3 \\
3 & 1 & -1
\end{array}\right) \text {. }
$$

При вычислении векторов двойственного базиса приходится вычислять матрицу $W^{-1}$, обратную матрице Вандермонда $(W)_{i j}=\lambda_{j}^{i-1}$. Приведем явные формулы для ее элементов в общем случае - для матрицы размера $n \times n$ и когда величины $\lambda_{j}$ удовлетворяют произвольному уравнению $f_{n} \lambda_{j}^{n}+\cdots+f_{1} \lambda_{j}+f_{0}=0$ с комплексньми коэффициентами $f_{j}$ :

$$
\left(W^{-1}\right)_{j i}=\frac{f_{n} \lambda_{j}^{n-i}+\cdots+f_{i+1} \lambda_{j}+f_{i}}{n f_{n} \lambda_{j}^{n-1}+\cdots+2 f_{2} \lambda_{j}+f_{1}}, \quad j, i=1, \ldots, n .
$$


Из формулы (5) видно, что вектор-столбцы двойственного к $L_{j}$ базиса $M_{i}$ пропорциональны строкам обратной к матрице $L$ матрицы $L^{-1}=W^{-1} B_{1}^{-1}$. Отыскав последнюю матрицу с помощью формулы (15), введем векторы $M_{i}$ таким образом, чтобы их первые компоненты были равны 1. Составленную из этих вектор-столбцов матрицу обозначим $M$. Имеем

$$
M_{i}=\left(1, \frac{3 \lambda_{i}^{2}+26 \lambda_{i}+13}{11}, \frac{8 \lambda_{i}^{2}+73 \lambda_{i}+53}{11}\right), \quad i=1,2,3
$$

т.е.

$$
\begin{aligned}
& M_{1} \approx(1,0.2632362140,-0.3843185164), \\
& M_{2} \approx(1,-0.6849428344,-0.4528201243), \\
& M_{3} \approx(1,1.5126157113,5.7462295498) .
\end{aligned}
$$

Для этих векторов все константы $\psi_{i}$ в формуле (5) положительны.

Определим кубические формы $g_{4}(X)$ и $g_{4}^{*}(U)$ формулами

$$
\begin{aligned}
& g_{4}(X)=\prod_{i=1}^{3}\left\langle L E_{i}, X\right\rangle=\operatorname{Norm}\left(\sum_{j=1}^{3}\left(L_{1}\right)_{j} x_{j}\right)=\operatorname{Norm}\left(\left\langle L_{1}, X\right\rangle\right), \quad X \in \mathbb{R}^{3}, \\
& g_{4}^{*}(U)=\prod_{i=1}^{3}\left\langle U, M E_{i}\right\rangle=\operatorname{Norm}\left(\sum_{j=1}^{3} u_{j}\left(M_{1}\right)_{j}\right)=\operatorname{Norm}\left(\left\langle U, M_{1}\right\rangle\right), \quad U \in \mathbb{R}_{*}^{3}
\end{aligned}
$$

( $E_{i}-i$-й базисньй вектор). Наряду с формами $g_{4}$ и $g_{4}^{*}$ введем пропорциональные им формы $\widetilde{g}_{4}(X)=5 g_{4}(X), \widetilde{g}_{4}^{*}(U)=11 g_{4}^{*}(U)$. Преимущество последних в том, что все принимаемые ими в целых точках значения - взаимно простые в совокупности целые числа, а не просто рациональные. Определители матриц $L$ и $M$ равны $\operatorname{det} L=-63 / 5$, $\operatorname{det} M=-63 / 11$, а минимумы модулей форм $\widetilde{g}_{4}$ и $\widetilde{g}_{4}^{*}$ суть 5 и 3 соответственно. Поэтому величины $(1)$ равны $\mu\left(g_{4}\right)=\mu\left(\widetilde{g}_{4}\right)=5 / 63, \mu\left(g_{4}^{*}\right)=\mu\left(\widetilde{g}_{4}^{*}\right)=1 / 21$. Пары форм $g_{4}$ и $g_{4}^{*}$, так же как и $\widetilde{g}_{4}$ и $\widetilde{g}_{4}^{*}$, будем называть взаимно сопряженными.

Пусть линейный оператор $D$ в $\mathbb{R}^{3}$ собственньми векторами имеет векторы $M_{i}$, а собственньпи значениями - некоторые числа $\delta_{i}$. То есть, $D M=M \Delta$, где матрица $\Delta$ диагональная: $\Delta=\operatorname{diag}\left\{\delta_{1}, \delta_{2}, \delta_{3}\right\}$. Тогда $\left\langle L_{j}, D X\right\rangle=\delta_{j}\left\langle L_{j}, X\right\rangle$ и преобразование $Y=D X$ переводит плоскости $\mathcal{L}_{i}$ в себя, а полупространства, на которые они делят $\mathbb{R}^{3},-$ в себя или в дополнительные полупространства. Следовательно, $D$ переводит октанты $\mathscr{O}_{\Sigma}$ в другие такие октанты. Поскольку $D^{*} L=L \Delta$, то поверхность уровня $g_{4}=c$ формы $g_{4}$ при этом преобразовании переходит в поверхность уровня $c \operatorname{det} D$, ибо

$$
g_{4}(D X)=\prod_{i=1}^{3}\left\langle L E_{i}, D X\right\rangle=\prod_{i=1}^{3}\left\langle D^{*} L E_{i}, X\right\rangle=\prod_{i=1}^{3}\left\langle L E_{i}, X\right\rangle \delta_{i}=g_{4}(X) \operatorname{det} D
$$

Если определитель матрищы $D$ равен \pm 1 , то относительно преобразования $Y=D X$ инвариантно абсолютное значение формы $g_{4}$. Итак, преобразования, одновременно являющиеся и унимодулярными $(D \in S L(3, \mathbb{Z}))$, и операторами сжатия-растяжения вдоль векторов $M_{i}$, переводят многогранники Клейна $\partial K_{\Sigma}$ друг в друга. Форма $g_{4}^{*}$ инвариантна относительно действия оператора $D^{*}$ в $\mathbb{R}_{*}^{3}$ (с транспонированной матрицей). 
Имеются отображения, композицией которых многогранник Клейна любого октанта переводится в многогранник Клейна любого другого октанта:

$$
\begin{gathered}
-E: \partial K_{\sigma_{1} \sigma_{2} \sigma_{3}} \rightarrow \partial K_{-\sigma_{1}-\sigma_{2}-\sigma_{3},}, \quad A_{1}^{*}: \partial K_{\sigma_{1} \sigma_{2} \sigma_{3}} \rightarrow \partial K_{-\sigma_{1}-\sigma_{2} \sigma_{3}} \\
A_{1}^{*}+E: \partial K_{\sigma_{1} \sigma_{2} \sigma_{3}} \rightarrow \partial K_{-\sigma_{1} \sigma_{2} \sigma_{3}} .
\end{gathered}
$$

Поэтому восемь многогранников Клейна $\partial K_{\Sigma}$ изоморфны между собой и достаточно вычислить только один из них.

Методом, описанным в п. 2, были вычислены целочисленные точки многогранников Клейна форм $g_{4}$ и $g_{4}^{*}$ (или, что то же самое, многогранников Клейна форм $\widetilde{g}_{4}$ и $\widetilde{g}_{4}^{*}$ ), лежашие в октантах $\mathscr{O}_{+++}$и $\mathscr{O}_{+++}^{*}$ соответственно. Результаты приведены в табл. 1 и 2. Оказалось, что в вершинах многогранника $\partial K_{+++}$форма $\widetilde{g}_{4}(X)$ принимает значения 5 , 9 , во внутренних точках ребер - значения 23 и во внутренних точках граней - $21,33,49$. Для многогранника $\partial K_{+++}^{*}$ значения формы $\widetilde{g}_{4}^{*}$ в вершинах суть $3,7,11$ и во внутренних точках граней - значения 25. В указанных октантах были также найдены не лежащие на многогранниках точки относительных минимумов. Их координаты и значения в них форм даны в табл. 3 и 4. Обобщенные данные соответствия сопряженных точек из сопряженных октантов приведены в табл. 5: указаны значения форм $\widetilde{g}_{4}$ и $\widetilde{g}_{4}^{*}$ в точках многогранника и значения, принимаемые сопряженными формами (соответственно $\widetilde{g}_{4}^{*}$ и $\left.\widetilde{g}_{4}\right)$ в точках, сопряженных к этим точкам. Эти данные свидетельствуют о том, что многогранники $\partial K_{+++}$и $\partial K_{+++}^{*}$ по структуре не похожи один на другой.

Введя логарифмические координаты $n_{i}(X)$ точки $X$, можно наглядно представить многогранник Клейна. Зафиксируем октант $\mathscr{O}_{\Sigma}$ и для его внутренних точек $X$ положим $l_{i}=l_{i}(X)=\ln \left|\left\langle L_{i}, X\right\rangle\right|, i=1,2,3$, и

$$
n_{1}=\frac{l_{1}+l_{2}+l_{3}}{\sqrt{3}}, \quad n_{2}=\frac{-l_{1}+2 l_{2}-l_{3}}{\sqrt{6}}, \quad n_{3}=\frac{l_{1}-l_{3}}{\sqrt{2}} .
$$

Координаты $n_{2}, n_{3}$ - это логарифмические координаты центральной проекции точки на поверхность уровня 1 функции $g_{4}$, а координата $n_{1}$ параметризует поверхности $g_{4}=$ const. В двойственном пространстве координаты $n_{i}$ введем тем же ортогональным преобразованием (18) с той разнищей, что $l_{i}=l_{i}(U)=\ln \left|\left\langle U, M_{i}\right\rangle\right|, i=1,2,3$.

На рис. 1 и 2 в этих координатах изображены проекции многогранников Клейна $\partial K_{+++}$и $\partial K_{+++}^{*}$ соответственно и точек относительных минимумов, не лежащих на них (чтобы отличать их, последние не закрашены). Рядом с точками подписаны значения, принимаемые в них формами $\widetilde{g}_{4}$ и $\widetilde{g}_{4}^{*}$ соответственно.

Введем обозначения для некоторых треугольных и четырехугольньх областей $\Gamma_{j}^{(2)}$ и $\Gamma_{j}^{(2) *}-$ граней или частей граней многогранников $\partial K_{+++}$и $\partial K_{+++}^{*}$ соответственно:

$$
\begin{array}{ll}
\Gamma_{1}^{(2)}=\{(1,0,1),(1,0,0),(2,-1,0)\}, & \rho=1, \\
\Gamma_{2}^{(2)}=\{(13,3,-5),(39,10,-15),(5,0,-2)\}, & \rho=1, \\
\Gamma_{3}^{(2)}=\{(10,3,-2),(30,8,-11),(3,1,0)\}, & \rho=1, \\
\Gamma_{4}^{(2)}=\{(1,0,1),(1,1,4),(1,0,0)\}, & \rho=1, \\
\Gamma_{5}^{(2)}=\{(1,0,1),(2,-1,0),(1,1,4)\}, & \rho=1,
\end{array}
$$




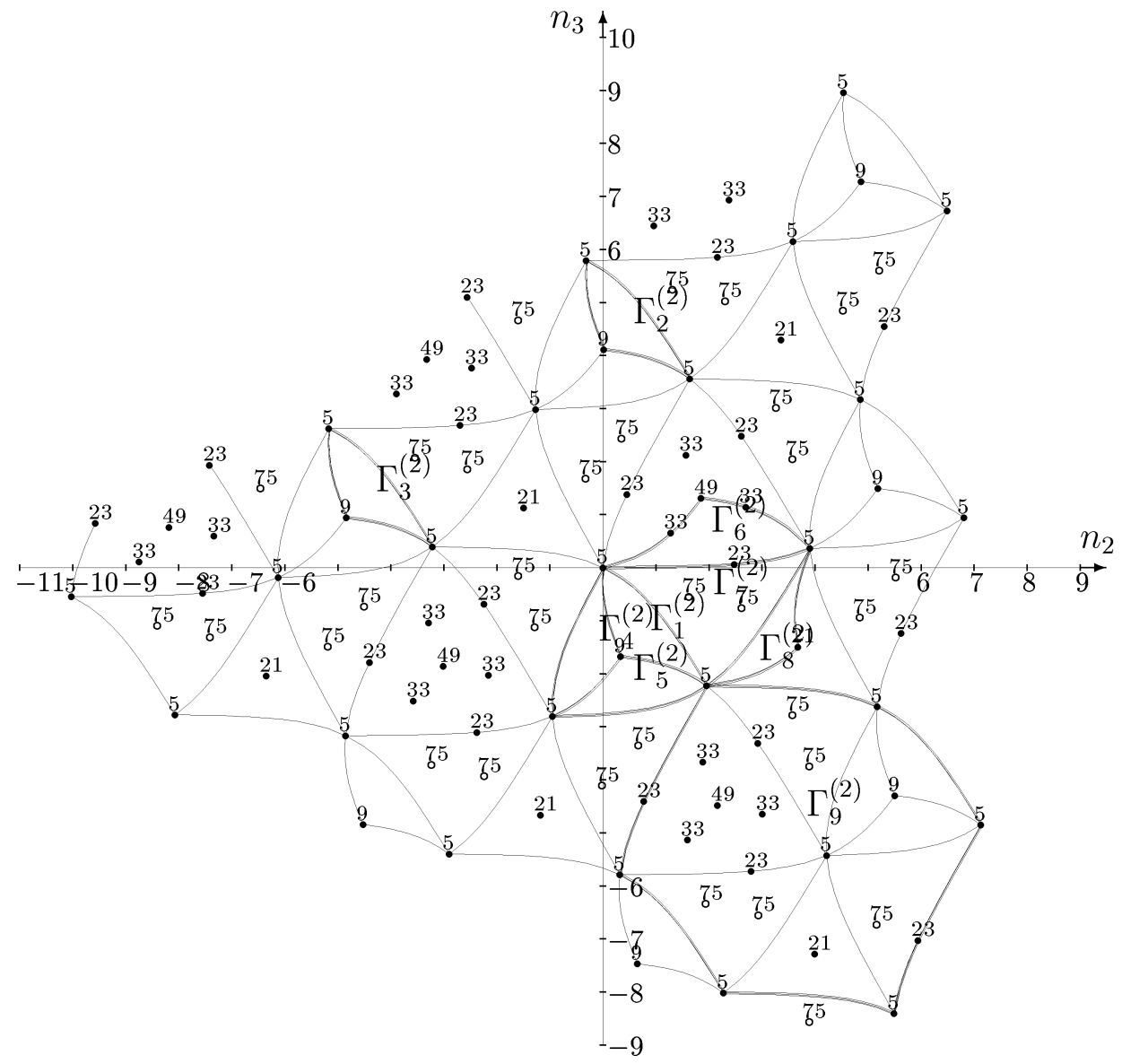

Рис. 1. Проекция многогранника Клейна $\partial K_{+++}$для формы $g_{4}$ в логарифмических координатах.

$$
\begin{array}{rlrl}
\Gamma_{6}^{(2)} & =\{(1,0,0),(5,-2,-2),(9,-6,-4)\}, & & \rho=1, \\
\Gamma_{7}^{(2)} & =\{(1,0,0),(9,-6,-4),(2,-1,0)\}, & & \rho=2, \\
\Gamma_{8}^{(2)} & =\{(2,-1,0),(9,-6,-4),(12,-8,-5)\}, & & \rho=1, \\
\Gamma_{1}^{(2) *} & =\{(1,0,2),(3,-1,7),(9,-2,22),(17,-3,42)\}, & \rho=1, \\
\Gamma_{2}^{(2) *} & =\{(9,17,-6),(2,3,-1),(1,1,0),(3,5,-1)\}, & & \rho=1, \\
\Gamma_{3}^{(2) *} & =\{(8,13,-2),(5,2,8),(12,-1,28),(24,0,53)\}, & \rho=1, \\
\Gamma_{4}^{(2) *} & =\{(16,-5,38) / 3,(9,-2,22),(3,-1,7)\}, & & \rho=1, \\
\Gamma_{5}^{(2) *} & =\{(16,-5,38) / 3,(3,-1,7),(4,-2,9)\}, & & \rho=1, \\
\Gamma_{6}^{(2) *} & =\{(16,-5,38) / 3,(4,-2,9),(9,-2,22)\}, & \rho & =1, \\
\Gamma_{7}^{(2) *} & =\{(1,0,2),(17,-3,42),(5,0,11)\}, & & \rho=3 .
\end{array}
$$

В правом столбце здесь приведены расстояния $\rho$ граней, частями которых являются эти 


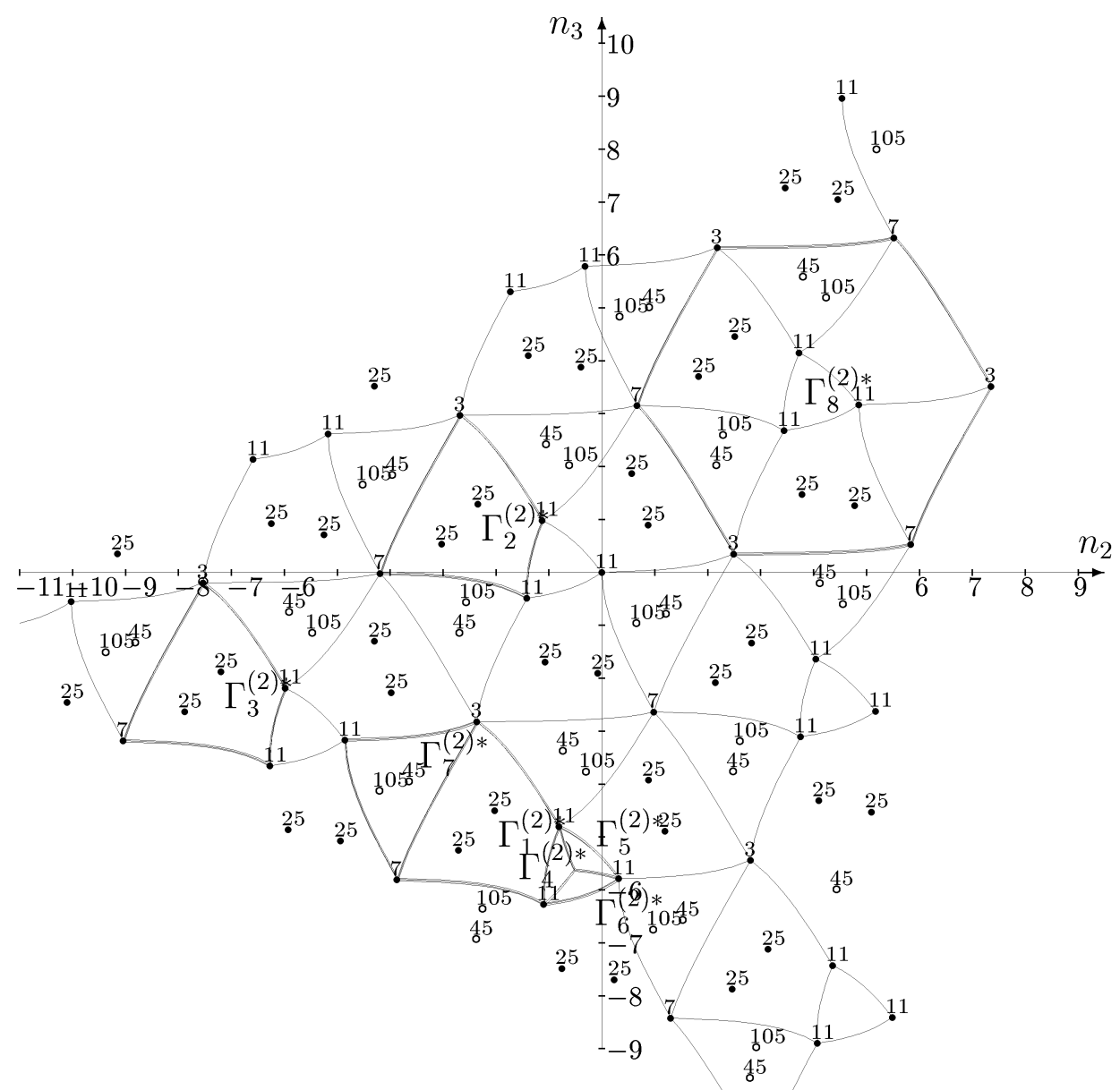

Рис. 2. Проекция сопряженного многогранника Клейна $\partial K_{+++}^{*}$ для формы $g_{4}^{*}$ в логарифмических координатах.

области. Здесь полныеграни суть $\Gamma_{1}^{(2)}-\Gamma_{5}^{(2)}, \Gamma_{7}^{(2)}$ и $\Gamma_{1}^{(2) *}-\Gamma_{3}^{(2) *}, \Gamma_{7}^{(2) *}$. Одна из вершин треугольников $\Gamma_{4}^{(2) *}, \Gamma_{5}^{(2) *}, \Gamma_{6}^{(2) *}$ - нецелая точка.

Найдем линейные отображения $C_{1}, C_{2}$, сопряженные преобразования к которым переводят друг в друга грани многогранника Клейна $\partial K_{+++}$. Они задаются своим действием в трех точках. Приняв за эти точки вершины граней $\Gamma_{1}^{(2)}, \Gamma_{2}^{(2)}$ и $\Gamma_{1}^{(2)}, \Gamma_{3}^{(2)}$ соответственно, получаем линейные системы и определяем $C_{i}^{*}$ :

$$
\begin{array}{rlr}
C_{1}^{*}\left(\begin{array}{ccc}
1 & 1 & 2 \\
0 & 0 & -1 \\
1 & 0 & 0
\end{array}\right) & =\left(\begin{array}{ccc}
13 & 39 & 5 \\
3 & 10 & 0 \\
-5 & -15 & -2
\end{array}\right), & C_{1}^{*}=\left(\begin{array}{ccc}
39 & 73 & -26 \\
10 & 20 & -7 \\
-15 & -28 & 10
\end{array}\right), \\
C_{2}^{*}\left(\begin{array}{ccc}
1 & 1 & 2 \\
0 & 0 & -1 \\
1 & 0 & 0
\end{array}\right) & =\left(\begin{array}{ccc}
10 & 30 & 3 \\
3 & 8 & 1 \\
-2 & -11 & 0
\end{array}\right), & C_{2}^{*}=\left(\begin{array}{ccc}
30 & 57 & -20 \\
8 & 15 & -5 \\
-11 & -22 & 9
\end{array}\right) .
\end{array}
$$


Можно проверить, что преобразования $C_{1}, C_{2}$ переводят грани $\Gamma_{1}^{(2) *}$ в грани $\Gamma_{2}^{(2) *}$ и $\Gamma_{3}^{(2) *}$ соответственно.

Операторы $C_{1}^{*}$ и $C_{2}^{*}$ задают автоморфизмы формы $g_{4}(X)$, а $C_{1}$ и $C_{2}$ - автоморфизмы формы $g_{4}^{*}(U)$. Для матрищ $C_{1}$ и $C_{2}$ (и сопряженных к ним $C_{1}^{*}, C_{2}^{*}$ ) характеристические уравнения суть соответственно

$$
\nu^{3}-69 \nu^{2}+54 \nu-1=0, \quad \nu^{3}-54 \nu^{2}+69 \nu-1=0
$$

При этом видно, что для обратных преобразований верно другое уравнение из пары (19): для $C_{2}^{-1}-$ первое, и для $C_{1}^{-1}-$ второе. Поскольку все преобразования переводят октанты в себя, собственные векторы у операторов $C_{1}, C_{2}$ те же, что и у оператора $A_{1}$. Сказанное остается, естественно, справедливо и для транспонированных матрищ $C_{1}^{*}$ и $C_{2}^{*}$ и оператора $A_{1}^{*}$.

Установим одну удобную формулу. Пусть матрицы $P$ и $Q$ имеют один и тот же набор собственных векторов (вектор-столбцов): $P V_{i}=V_{i} p_{i}, Q V_{i}=V_{i} q_{i}, p_{i}, q_{i} \in \mathbb{C}, i=$ $1, \ldots, n$, причем числа $p_{i}$ различны. Найдем представление матрицы $Q$ в виде полинома от матрицы $P$

$$
Q=f_{Q, P}(P)=\sum_{j=1}^{n} k_{j} P^{j-1} .
$$

Применив представление (20) к векторам $V_{i}$, получим

$$
q_{i} V_{i}=Q V_{i}=\sum_{j=1}^{n} k_{j} P^{j-1} V_{i}=\sum_{j=1}^{n} k_{j} p_{i}^{j-1} V_{i}
$$

т.е. $q_{i}=\sum_{j=1}^{n} k_{j} p_{i}^{j-1}$. Зададим векторы $Q, K$ и матрицу Вандермонда $W_{P}$ формулами: $(Q)_{i}=q_{i},(K)_{i}=k_{i},\left(W_{P}\right)_{i j}=p_{i}^{j-1}, i, j=1, \ldots, n$. Теперь видно, что существование представления (20) сводится к разрешимости системы $W_{P} K=Q$. Так как $p_{i}$ различны, матрица Вандермонда обратима, и коэффициенты полинома равны

$$
k_{j}=\sum_{l=1}^{n}\left(W_{P}^{-1}\right)_{j l} q_{l}, \quad j=1, \ldots, n
$$

Формулы (20), (21) позволяют найти представление найденных выше матриц как полиномов от матрицы $A_{1}: C_{1}=A_{1}^{2}, C_{2}=\left(A_{1}+E\right)^{2}$.

Другие преобразования, сохраняющие октанты, это перестановки вершин некоторых треугольных граней многогранника. На рис. 1 для многогранника $\partial K_{+++}$такое преобразование $C_{0}^{*}$ вьглядит как поворот против часовой стрелки вокруг точки $(1,0,1)$. Из условия, что область $\Gamma_{4}^{(2)}$ переходит в $\Gamma_{5}^{(2)}$, получаем

$$
C_{0}^{*}\left(\begin{array}{ccc}
1 & 1 & 1 \\
0 & 0 & 1 \\
0 & 1 & 4
\end{array}\right)=\left(\begin{array}{ccc}
2 & 1 & 1 \\
-1 & 0 & 0 \\
0 & 1 & 0
\end{array}\right) ; \quad C_{0}^{*}=\left(\begin{array}{ccc}
2 & 3 & -1 \\
-1 & -3 & 1 \\
0 & -4 & 1
\end{array}\right)
$$

Куб этого преобразования равен тождественному преобразованию. Для многогранника $\partial K_{+++}^{*}$ аналогично действует преобразование $C_{0}$ с транспонированной матрицей. Матрица $C_{0}$ не коммутирует с $A_{1}$, не является многочленом от этой матрицы и поэтому 
существенно расширяет групш изоморфизмов многогранника Клейна, делая ее некоммутативной:

$$
C_{1}^{*} C_{0}^{*}=C_{0}^{*}\left(C_{1}^{*}\right)^{-1} C_{2}^{*}, \quad C_{2}^{*} C_{0}^{*}=C_{0}^{*}\left(C_{1}^{*}\right)^{-1} .
$$

Все вершины $X$ одной полости (лежашей в октанте $\mathscr{O}_{+++}$) многогранника Клейна $\partial K$, значения формы $g_{4}(X)$ в которых одинаковы, можно получить из одной такой вершины $X_{0}$ по формуле $X=\left(C_{1}^{*}\right)^{i}\left(C_{2}^{*}\right)^{j} X_{0}, i, j \in \mathbb{Z}$. При этом некоторая фиксированная "соседняя" с $X_{0}$ вершина $X_{0}^{\prime}$ отобразится в одну из трех вершин "соседних" с $X$. Предварительньм “поворотом" $\left(V^{*}\right)^{m}, m=0,1,2$, вокруг точки $X_{0}$ можно добиться, чтобы $X_{0}^{\prime}$ отобразилась в какую-то конкретную “соседнюю” с $X$ вершину $X^{\prime}$ :

$$
\begin{aligned}
X & =\left(C_{1}^{*}\right)^{i}\left(C_{2}^{*}\right)^{j}\left(V^{*}\right)^{m} X_{0}, \quad i, j \in \mathbb{Z}, \quad m=0,1,2, \\
X^{\prime} & =\left(C_{1}^{*}\right)^{i}\left(C_{2}^{*}\right)^{j}\left(V^{*}\right)^{m} X_{0}^{\prime} .
\end{aligned}
$$

Отметим, что для точек $X$ - вершин треугольника $\Gamma_{1}^{(2)} \cup \Gamma_{4}^{(2)} \cup \Gamma_{5}^{(2)}$ - преобразованием $V^{*}$ будет преобразование $C_{0}^{*}$ или $\left(C_{0}^{*}\right)^{2}$. Теперь осталось заметить, что многогранники Клейна разных полостей переводятся друг в друга преобразованиями (17).

Пользуясь тем, что единственньй автоморфизм многогранников Клейна $\cup_{\Sigma} \partial K_{\Sigma}$, имеющий ненулевую целочисленную неподвижную точку, это тождественное преобразование, получаем представление произвольного автоморфизма формы $g_{4}$ в виде произведения

$$
\left(A_{1}^{*}\right)^{k}\left(A_{1}^{*}+E\right)^{l}\left(C_{0}^{*}\right)^{m}(-E)^{n}, \quad k, l \in \mathbb{Z}, \quad m=0,1,2, \quad n=0,1 .
$$

В качестве фундаментальной области многогранника Клейна относительно групшы (22) может быть взята область $H$ из четырех треугольников $\Gamma_{1}^{(2)}, \Gamma_{6}^{(2)}, \Gamma_{7}^{(2)}$ и $\Gamma_{8}^{(2)}$ расстояний 1, 1, 2 и 1 соответственно. Итак, доказана

Теорема 1. Для формы $g_{4}$ группа автоморфизмов $G\left(g_{4}\right)$ имеет две образующих бесконечного порядка $A_{1}^{*} u A_{1}^{*}+E$ u две образующих $C_{0} u-E$ порядков 3 и 2 соответственно. Фундаментальная область $H$ всех многогранников Клейна $\bigcup_{\Sigma} \partial K_{\Sigma}$ состоит из четырех треугольников $\Gamma_{1}^{(2)}, \Gamma_{6}^{(2)}, \Gamma_{7}^{(2)}$ и $\Gamma_{8}^{(2)}$ расстояний 1 , 1,2 и 1 соответственно. Для формы $g_{4}^{*}$ образуюшими группы автоморфизмов $G\left(g_{4}^{*}\right)$ служат преобразования, сопряженные $\kappa$ образующим группы $G\left(g_{4}\right)$. Фундаментальная область $H_{*}$ всех многогранников Клейна $\bigcup_{\Sigma} \partial K_{\Sigma}^{*}$ состоит из четырехугольника $\Gamma_{1}^{(2) *}$ расстояния 1 и двух треугольников $\Gamma_{4}^{(2) *} u \Gamma_{7}^{(2) *}$ расстояний $1 u 3$.

Фундаментальные области будут состоять не из частей граней, а из целых граней, если ограничиться рассмотрением лишь коммутативных подгрупш групп автоморфизмов многогранника Клейна (подгруппы преобразований, для которых в формуле (22) $m=0)$. На рисунках 1 и 2 такие фундаментальные области обозначены символами $\Gamma_{9}^{(2)}$ и $\Gamma_{8}^{(2) *}$ соответственно. По одной из вершин изображенных областей (точек $(123,-84,-55)$ и $(37,-108,22))$ нет в табл. 1 и 2 : они добавлены, чтобы указанные области не заходили на изображенные ранее.

5. Разложения по матричным алгоритмам. Был исследован вопрос, улавливают ли структуру многогранников Клейна различные многомерные матричные обобщения разложений в цепные дроби. Вычислялись разложения векторов по алгоритмам Эйлера, Якоби (4 варианта), Пуанкаре, Бруна, Брюно и Парусникова (см. [15], [16]). 
При этом в [15], [16] алгоритм Брюно описан неверно. Поэтому приводим здесь его правильное описание. Для тройки чисел $\alpha \geqslant \beta \geqslant \gamma \geqslant 0$ вьгисляются $a=[\alpha / \beta], b=[\beta / \gamma]$, $c=[(\alpha-a \beta) / \gamma]\left([\right.$.$] - целая часть) и делаются преобразования \alpha^{\prime}=\alpha-a \beta-c \gamma$, $\beta^{\prime}=\beta-b \gamma, \gamma^{\prime}=\gamma$. Числа $\alpha^{\prime}, \beta^{\prime}, \gamma^{\prime}$ упорядочиваются и процедура повторяется и т.д. В настоящей работе использовался ускоренный алгоритм Бруна. Шаги алгоритма Бруна, на которых не происходит переупорядочения компонент векторов, в нем опущены, т.е. шаг алгоритма состоит в получении чисел $\alpha^{\prime}=\alpha-[\alpha / \beta] \beta, \beta^{\prime}=\beta, \gamma^{\prime}=-\gamma$ и их упорядочения.

Рассматривались два начальных вектора $L_{3}^{\prime}$ и $M_{3}$. В первом случае разлагался вектор $L_{3}^{\prime}$, полученный из вектора $L_{3}$ в $(11)$ изменением знака его второй компоненты. При этом для “подходящих дробей” $U^{\prime}$ вектора $L_{3}^{\prime}$ вычислялись значения форм $\left\langle U^{\prime}, M_{i}^{\prime}\right\rangle$ и $\widetilde{g}_{4}^{*}\left(U^{\prime}\right)=11\left\langle U^{\prime}, M_{1}^{\prime}\right\rangle\left\langle U^{\prime}, M_{2}^{\prime}\right\rangle\left\langle U^{\prime}, M_{3}^{\prime}\right\rangle$, где векторы $M_{i}^{\prime}$ образованы из $M_{i}$ в $(16)$ заменой знака второй компоненты.

Обозначим с помощью дополнительного индекса $k$ вектор остаток цепной дроби, получающийся на $k$-м шаге матричного алгоритма: $L_{3,-1}^{\prime}=L_{3}^{\prime}$. Соседние остатки связаны друг с другом соотношениями $L_{3, k}^{\prime}=Q_{k} L_{3, k-1}^{\prime}$ с унимодулярными матрищами $Q_{k}$. Введем матрицу $\widetilde{Q}_{0}$ такую, что компоненты $\widetilde{Q}_{0} L_{3}^{\prime}-$ это упорядоченные в порядке убывания компоненты вектора $L_{3}^{\prime}$, и положим для всех алгоритмов, кроме алгоритмов Якоби, $Q_{0}=\widetilde{Q}_{0}$. Для вариантов алгоритмов Якоби введем матрищы

$$
Q^{(123)}=E, \quad Q^{(132)}=\left(\begin{array}{lll}
1 & 0 & 0 \\
0 & 0 & 1 \\
0 & 1 & 0
\end{array}\right), \quad Q^{(213)}=\left(\begin{array}{lll}
0 & 1 & 0 \\
1 & 0 & 0 \\
0 & 0 & 1
\end{array}\right), \quad Q^{(312)}=\left(\begin{array}{lll}
0 & 0 & 1 \\
1 & 0 & 0 \\
0 & 1 & 0
\end{array}\right)
$$

и положим $Q_{0}=Q^{(\kappa)} \widetilde{Q}_{0}, \kappa=$ "123", "132", "213", "312".

В табл. 6 представлено разложение вектора $L_{3}^{\prime}$ по единственному периодическому алгоритму из исследованных - алгоритму Бруна. (Разложения $L_{3}^{\prime}$ по другим алгоритмам приведены в [16].) Столбцы таблицы содержат: $k$ - номер шага алгоритма, вектор остатка $L_{k}$, матрицу $Q_{k}$, матрицу $N_{k}=\left(M_{k} \ldots M_{0} M_{-1}\right)^{-1}$, столбец значений формы $\widetilde{g}_{4}$ на подходящих дробя $N_{k} E_{j}, j=1,2,3$, и нормированньй вектор остатка $L_{k}^{\prime} /\left(L_{k}^{\prime}\right)_{1}$. Кроме алгоритма Бруна, все рассмотренные алгоритмы непериодичны. Выявленньй период длины 7 ускоренного алгоритма Бруна (длины 10 для оригинального алгоритма) задается матрищей

$$
T_{1}=\left(\begin{array}{ccc}
4 & -6 & 23 \\
1 & -1 & 4 \\
10 & -15 & 57
\end{array}\right)=7 \widehat{A}_{1}^{2}+64 \widehat{A}_{1}+51 E=\widehat{A}_{1}^{-2}\left(\widehat{A}_{1}+E\right)
$$

где матрица $\widehat{A_{1}}$ отличается от $A_{1}$ заменой знаков вторых строк и столбцов

$$
\widehat{A_{1}}=\left(\begin{array}{ccc}
-5 & 1 & 2 \\
8 & -3 & -3 \\
3 & -1 & -1
\end{array}\right)
$$

Алгоритм Брюно, хотя его период не найден, остается на многогранниках Клейна. Алгоритмы Якоби (132) и (312) на некоторых шагах сходят с многогранников Клейна, но потом вновь возвращаются на многогранники. "Подходящие дроби", вычисленные по 
остальным алгоритмам, сходят с многогранников Клейна уже на втором - третьем шагах.

Во втором случае разлагался положительньй вектор $M_{3} \in \mathbb{R}_{*}^{3}$ из (16). В подходящих дробях $X$ к нему вычислялись значения форм $\left\langle L_{i}, X\right\rangle$ и $\widetilde{g}_{4}(X)$. Для алгоритма Брюно был найден период длины 3:

$$
T_{2}=\left(\begin{array}{ccc}
4 & -1 & 10 \\
6 & -1 & 15 \\
23 & -4 & 57
\end{array}\right)=7\left(A_{1}^{*}\right)^{2}+64 A_{1}^{*}+51 E=\left(A_{1}^{*}\right)^{-2}\left(A_{1}^{*}+E\right)
$$

где матрица $A_{1}^{*}$ - транспонированная матрица (14). В табл. 7 , организованной так же, как табл. 6, представлено разложение $M_{3}$ по алгоритму Брюно. (Разложения $M_{3}$ по всем алгоритмам приведены в [16].) Алгоритмы Якоби (132) и (312) и алгоритм Бруна остаются на многогранниках Клейна. Их периоды не были обнаружены. Остальные алгоритмы сходят с многогранников Клейна на втором - четвертом шагах.

Мы видим, что при разложении векторов из сопряженных пространств (которые в рассматриваемом случае различны) “качество” этих алгоритмов разложения меняетcя.

Автор благодарит А. Д. Брюно за постановку задачи и полезные замечания.

\section{Приложение}

ТАБлицА 1 . Точки $X=\left(x_{1}, x_{2}, x_{3}\right)$ с $\left|x_{2}\right|,\left|x_{3}\right| \leqslant 100$ многогранника Клейна $\partial K_{+++}$формы $\widetilde{g}_{4}$.

\begin{tabular}{|c|c|c|c|c|c|c|c|}
\hline$X$ & $\widetilde{g}_{4}(X)$ & $X$ & $\widetilde{g}_{4}(X)$ & $X$ & $\widetilde{g}_{4}(X)$ & $X$ & $\widetilde{g}_{4}(X)$ \\
\hline$(1,0,0)$ & 5 & $(39,10,-15)$ & 5 & $(3,0,-1)$ & 23 & $(4,-1,3)$ & 33 \\
$(1,1,4)$ & 5 & $(65,3,-26)$ & 5 & $(3,2,10)$ & 23 & $(4,5,18)$ & 33 \\
$(2,-1,0)$ & 5 & $(78,-49,-35)$ & 5 & $(3,4,15)$ & 23 & $(5,-1,-2)$ & 33 \\
$(3,1,0)$ & 5 & $(95,-65,-43)$ & 5 & $(4,4,13)$ & 23 & $(5,2,13)$ & 33 \\
$(4,5,20)$ & 5 & $(1,0,1)$ & 9 & $(5,-3,-2)$ & 23 & $(7,-4,-3)$ & 33 \\
$(5,0,-2)$ & 5 & $(10,3,-2)$ & 9 & $(7,-4,-1)$ & 23 & $(9,-4,2)$ & 33 \\
$(5,7,26)$ & 5 & $(13,3,-5)$ & 9 & $(7,-3,-3)$ & 23 & $(34,11,-3)$ & 33 \\
$(7,3,3)$ & 5 & $(13,18,70)$ & 9 & $(8,-1,9)$ & 23 & $(42,11,-16)$ & 33 \\
$(8,2,-3)$ & 5 & $(16,24,91)$ & 9 & $(18,9,14)$ & 23 & $(45,17,8)$ & 33 \\
$(9,-6,-4)$ & 5 & $(31,-21,-14)$ & 9 & $(19,5,-7)$ & 23 & $(53,14,-20)$ & 33 \\
$(10,10,43)$ & 5 & $(40,-27,-17)$ & 9 & $(28,4,-11)$ & 23 & $(59,11,-23)$ & 33 \\
$(10,15,57)$ & 5 & $(40,-12,-17)$ & 9 & $(49,-31,-22)$ & 23 & $(70,17,-27)$ & 33 \\
$(12,-7,-2)$ & 5 & $(61,-21,31)$ & 9 & $(60,-41,-27)$ & 23 & $(3,3,10)$ & 49 \\
$(15,20,73)$ & 5 & $(4,1,-1)$ & 21 & $(77,21,-26)$ & 23 & $(5,-2,-2)$ & 49 \\
$(17,-2,-7)$ & 5 & $(5,7,27)$ & 21 & $(79,-51,-27)$ & 23 & $(6,-1,6)$ & 49 \\
$(20,-13,-9)$ & 5 & $(9,10,34)$ & 21 & $(88,27,-15)$ & 23 & $(61,19,-9)$ & 49 \\
$(25,-17,-11)$ & 5 & $(12,-8,-5)$ & 21 & $(99,26,-38)$ & 23 & $(76,20,-29)$ & 49 \\
$(29,15,25)$ & 5 & $(14,-5,-6)$ & 21 & $(2,2,7)$ & 33 & & \\
$(30,8,-11)$ & 5 & $(19,-5,14)$ & 21 & $(3,-1,-1)$ & 33 & & \\
$(35,-18,1)$ & 5 & $(2,1,2)$ & 23 & $(3,2,5)$ & 33 & & \\
\hline
\end{tabular}


ТАБЛИџА 2. Точки $U=\left(u_{1}, u_{2}, u_{3}\right) \mathrm{c}\left|u_{2}\right|,\left|u_{3}\right| \leqslant 100$ многогранника Клейна $\partial K_{+++}^{*}$ формы $\widetilde{g}_{4}^{*}$.

\begin{tabular}{|c|c|c|c|c|c|c|c|}
\hline$U$ & $\widetilde{g}_{4}(U)$ & $U$ & $\widetilde{g}_{4}(U)$ & $U$ & $\widetilde{g}_{4}(U)$ & $U$ & $\widetilde{g}_{4}(U)$ \\
\hline$(1,0,2)$ & 3 & $(5,2,8)$ & 11 & $(1,0,1)$ & 25 & $(14,-40,9)$ & 25 \\
$(2,-5,1)$ & 3 & $(7,-16,3)$ & 11 & $(2,-3,3)$ & 25 & $(14,25,-9)$ & 25 \\
$(3,-7,3)$ & 3 & $(8,-23,5)$ & 11 & $(2,-1,0)$ & 25 & $(17,3,33)$ & 25 \\
$(8,13,-2)$ & 3 & $(9,-2,22)$ & 11 & $(3,-8,2)$ & 25 & $(19,-5,46)$ & 25 \\
$(9,17,-6)$ & 3 & $(10,-29,6)$ & 11 & $(3,-4,5)$ & 25 & $(19,35,-11)$ & 25 \\
$(13,18,-7)$ & 3 & $(10,-18,3)$ & 11 & $(3,1,5)$ & 25 & $(24,-69,14)$ & 25 \\
$(37,-15,86)$ & 3 & $(12,-1,28)$ & 11 & $(3,2,-1)$ & 25 & $(24,-5,59)$ & 25 \\
$(1,-2,1)$ & 7 & $(15,-38,13)$ & 11 & $(3,3,2)$ & 25 & $(24,-4,59)$ & 25 \\
$(3,5,-1)$ & 7 & $(17,-31,23)$ & 11 & $(4,7,-2)$ & 25 & $(24,45,-16)$ & 25 \\
$(4,5,-2)$ & 7 & $(20,-55,11)$ & 11 & $(5,-14,3)$ & 25 & $(31,-90,19)$ & 25 \\
$(14,-5,33)$ & 7 & $(25,-73,15)$ & 11 & $(5,-1,12)$ & 25 & $(31,-5,76)$ & 25 \\
$(17,-3,42)$ & 7 & $(29,43,-1)$ & 11 & $(5,9,-3)$ & 25 & $(36,-55,56)$ & 25 \\
$(24,0,53)$ & 7 & $(30,57,-20)$ & 11 & $(7,3,-2)$ & 25 & $(36,-20,-1)$ & 25 \\
$(33,-75,14)$ & 7 & $(35,-95,26)$ & 11 & $(10,-19,13)$ & 25 & $(39,10,71)$ & 25 \\
$(1,0,0)$ & 11 & $(38,72,-25)$ & 11 & $(10,1,-2)$ & 25 & $(39,35,-16)$ & 25 \\
$(1,1,0)$ & 11 & $(39,73,-26)$ & 11 & $(10,6,13)$ & 25 & $(43,76,-20)$ & 25 \\
$(2,3,-1)$ & 11 & $(44,53,17)$ & 11 & $(11,-30,6)$ & 25 & $(52,99,-35)$ & 25 \\
$(3,-1,7)$ & 11 & $(49,93,-33)$ & 11 & $(11,-2,27)$ & 25 & $(54,20,89)$ & 25 \\
$(4,-2,9)$ & 11 & $(65,52,-25)$ & 11 & $(11,20,-6)$ & 25 & & \\
$(5,0,11)$ & 11 & $(1,-1,1)$ & 25 & $(12,-12,23)$ & 25 & & \\
\hline
\end{tabular}

ТАБлицА 3. Точки относительных минимумов $X_{j}=\left(x_{1}, x_{2}, x_{3}\right) \in \mathscr{O}_{+++} \mathrm{c}$ $\left(\left|x_{2}\right|,\left|x_{3}\right| \leqslant 100\right.$ формы $\widetilde{g}_{4}$, не лежашие на $\partial K_{+++}$.

\begin{tabular}{|c|c|c|c|c|c|c|c|}
\hline$X$ & $\widetilde{g}_{4}(X)$ & $X$ & $\widetilde{g}_{4}(X)$ & $X$ & $\widetilde{g}_{4}(X)$ & $X$ & $\widetilde{g}_{4}(X)$ \\
\hline$(2,1,3)$ & 75 & $(8,-5,-3)$ & 75 & $(17,-11,-6)$ & 75 & $(36,7,73)$ & 75 \\
$(3,1,1)$ & 75 & $(8,1,-3)$ & 75 & $(18,-11,-8)$ & 75 & $(43,-23,-19)$ & 75 \\
$(3,1,7)$ & 75 & $(8,7,21)$ & 75 & $(20,16,45)$ & 75 & $(47,-32,-21)$ & 75 \\
$(4,-2,-1)$ & 75 & $(9,13,49)$ & 75 & $(22,-14,-7)$ & 75 & $(57,16,-17)$ & 75 \\
$(4,4,17)$ & 75 & $(11,7,36)$ & 75 & $(25,1,-10)$ & 75 & $(63,-38,-14)$ & 75 \\
$(6,1,-2)$ & 75 & $(15,1,25)$ & 75 & $(26,7,-9)$ & 75 & $(72,-41,-32)$ & 75 \\
$(7,4,8)$ & 75 & $(15,4,-5)$ & 75 & $(31,22,56)$ & 75 & $(73,19,-28)$ & 75 \\
$(7,10,38)$ & 75 & $(16,-8,-7)$ & 75 & $(36,7,-14)$ & 75 & $(82,-56,-37)$ & 75 \\
\hline
\end{tabular}


ТАБЛИцА 4. Точки относительных минимумов $U=\left(u_{1}, u_{2}, u_{3}\right)$ с $\left|u_{2}\right|,\left|u_{3}\right| \leqslant 100$ формы $\widetilde{g}_{4}^{*}$, не лежащие на $\partial K_{+++}^{*}$.

\begin{tabular}{|c|c|c|c|c|c|c|c|}
\hline$U$ & $\widetilde{g}_{4}(U)$ & $U$ & $\widetilde{g}_{4}(U)$ & $U$ & $\widetilde{g}_{4}(U)$ & $U$ & $\widetilde{g}_{4}(U)$ \\
\hline$(2,-4,1)$ & 45 & $(17,-49,10)$ & 45 & $(3,-2,6)$ & 105 & $(32,-93,19)$ & 105 \\
$(2,-1,4)$ & 45 & $(17,32,-11)$ & 45 & $(3,4,0)$ & 105 & $(32,-6,79)$ & 105 \\
$(2,2,1)$ & 45 & $(23,-64,16)$ & 45 & $(7,-19,5)$ & 105 & $(33,-56,9)$ & 105 \\
$(5,-13,4)$ & 45 & $(23,-19,1)$ & 45 & $(7,11,-4)$ & 105 & $(33,-29,66)$ & 105 \\
$(5,-7,1)$ & 45 & $(23,23,16)$ & 45 & $(8,-9,1)$ & 105 & $(33,58,-21)$ & 105 \\
$(8,-7,16)$ & 45 & $(23,38,-14)$ & 45 & $(8,9,4)$ & 105 & $(38,33,34)$ & 105 \\
$(8,-1,19)$ & 45 & $(35,-22,76)$ & 45 & $(13,-7,29)$ & 105 & $(95,-78,4)$ & 105 \\
$(8,11,1)$ & 45 & $(38,-7,94)$ & 45 & $(15,-2,36)$ & 105 & & \\
$(8,14,-5)$ & 45 & $(2,-3,1)$ & 105 & $(25,47,-16)$ & 105 & & \\
\hline
\end{tabular}

ТАБлицА 5. Значения форм $\widetilde{g}_{4}$ и $\widetilde{g}_{4}^{*}$ во взаимно сопряженных точках.

\begin{tabular}{|c|c|c|c|c|}
\hline Форма & $\begin{array}{c}\text { Значение } \\
\text { формы в точке }\end{array}$ & $\begin{array}{c}\text { Расположение } \\
\text { точки }\end{array}$ & $\begin{array}{c}\text { Сопряженная } \\
\text { форма }\end{array}$ & $\begin{array}{c}\text { Значения сопряженной формы } \\
\text { в сопряженных точках }\end{array}$ \\
\hline$\widetilde{g}_{4}$ & 5 & Вершина & $\widetilde{g}_{4}^{*}$ & $3,7,11,25$ \\
& 9 & Вершина & & 11 \\
& 21 & На грани & & 7 \\
& 23 & На ребре & & 3 \\
& 33 & На грани & & 3 \\
\hline$\widetilde{g}_{4}^{*}$ & 39 & На грани & & 3 \\
& 7 & Вершина & $\widetilde{g}_{4}$ & $5,23,33,49$ \\
& 11 & Вершина & & 5,9 \\
& 25 & Вершина & & 5 \\
\hline
\end{tabular}


ТАБлицА 6. Разложение вектора $L_{3}^{\prime}$ по ускоренному алгоритму Бруна.

\begin{tabular}{|c|c|c|c|c|c|c|c|c|c|}
\hline$k$ & $L_{k}^{\prime}$ & & $Q_{k}$ & & & $N_{k}$ & & $\widetilde{g}_{4}^{*}$ & $L_{k}^{\prime} /\left(L_{k}^{\prime}\right)_{1}$ \\
\hline \multirow[t]{3}{*}{0} & $.2479322 \mathrm{E}+1$ & 0 & 0 & 1 & 0 & 1 & 0 & 11 & 1 \\
\hline & 1 & 1 & 0 & 0 & 0 & 0 & 1 & 11 & .4033361 \\
\hline & .1791197 & 0 & 1 & 0 & 1 & 0 & 0 & 3 & .0722454 \\
\hline \multirow[t]{3}{*}{1} & 1. & 0 & 1 & 0 & 1 & 0 & 0 & 3 & 1. \\
\hline & .4793216 & 1 & -2 & 0 & 0 & 0 & 1 & 11 & .4793216 \\
\hline & .1791197 & 0 & 0 & 1 & 2 & 1 & 0 & 3 & .1791197 \\
\hline \multirow[t]{3}{*}{2} & .4793216 & 0 & 1 & 0 & 2 & 0 & 1 & -7 & 1. \\
\hline & .1791197 & 0 & 0 & 1 & 0 & 1 & 0 & 3 & .3736941 \\
\hline & $.4135671 \mathrm{E}-1$ & 1 & -2 & 0 & 5 & 0 & 2 & 3 & .0862818 \\
\hline \multirow[t]{3}{*}{3} & .1791197 & 0 & 1 & 0 & 4 & 2 & 1 & -11 & 1 \\
\hline & .1210823 & 1 & -2 & 0 & 1 & 0 & 0 & -7 & .6759855 \\
\hline & $.4135671 \mathrm{E}-1$ & 0 & 0 & 1 & 10 & 5 & 2 & 3 & .2308887 \\
\hline \multirow[t]{3}{*}{4} & .1210823 & 0 & 1 & 0 & 6 & 4 & 1 & 3 & 1 \\
\hline & $.5803737 \mathrm{E}-1$ & 1 & -1 & 0 & 1 & 1 & 0 & -11 & .4793216 \\
\hline & $.4135671 \mathrm{E}-1$ & 0 & 0 & 1 & 15 & 10 & 2 & 3 & .3415587 \\
\hline \multirow[t]{3}{*}{5} & $.5803737 \mathrm{E}-1$ & 0 & 1 & 0 & 16 & 1 & 6 & 25 & 1. \\
\hline & $.4135671 \mathrm{E}-1$ & 0 & 0 & 1 & 3 & 0 & 1 & 3 & .7125877 \\
\hline & $.5007566 \mathrm{E}-2$ & 1 & -2 & 0 & 40 & 2 & 15 & 3 & .0862818 \\
\hline \multirow[t]{3}{*}{6} & $.4135671 \mathrm{E}-1$ & 0 & 1 & 0 & 17 & 16 & 6 & 7 & 1. \\
\hline & $.1668066 \mathrm{E}-1$ & 1 & -1 & 0 & 3 & 3 & 1 & 25 & .4033361 \\
\hline & $.5007566 \mathrm{E}-2$ & 0 & 0 & 1 & 42 & 40 & 15 & 3 & .1210823 \\
\hline \multirow[t]{3}{*}{7} & $.1668066 \mathrm{E}-1$ & 0 & 1 & 0 & 50 & 17 & 6 & -3 & 1. \\
\hline & $.7995400 \mathrm{E}-2$ & 1 & -2 & 0 & 9 & 3 & 1 & 7 & .4793216 \\
\hline & $.5007566 \mathrm{E}-2$ & 0 & 0 & 1 & 124 & 42 & 15 & 3 & .3002020 \\
\hline \multirow[t]{3}{*}{8} & $.7995400 \mathrm{E}-2$ & 0 & 1 & 0 & 117 & 6 & 50 & 25 & 1. \\
\hline & $.5007566 \mathrm{E}-2$ & 0 & 0 & 1 & 21 & 1 & 9 & 3 & .6263059 \\
\hline & $.6898571 \mathrm{E}-3$ & 1 & -2 & 0 & 290 & 15 & 124 & -3 & .0862818 \\
\hline \multirow[t]{3}{*}{9} & $.5007566 \mathrm{E}-2$ & 0 & 1 & 0 & 123 & 117 & 50 & 7 & 1. \\
\hline & $.2987834 \mathrm{E}-2$ & 1 & -1 & 0 & 22 & 21 & 9 & 25 & .5966639 \\
\hline & $.6898571 \mathrm{E}-3$ & 0 & 0 & 1 & 305 & 290 & 124 & -3 & .1377630 \\
\hline \multirow[t]{3}{*}{10} & $.2987834 \mathrm{E}-2$ & 0 & 1 & 0 & 240 & 123 & 50 & 11 & 1 \\
\hline & $.2019732 \mathrm{E}-2$ & 1 & -1 & 0 & 43 & 22 & 9 & 7 & .6759855 \\
\hline & $.6898571 \mathrm{E}-3$ & 0 & 0 & 1 & 595 & 305 & 124 & -3 & .2308887 \\
\hline
\end{tabular}

$$
T_{1}=\left(\begin{array}{ccc}
4 & -6 & 23 \\
1 & -1 & 4 \\
10 & -15 & 57
\end{array}\right)
$$


ТАБлицА 7. Разложение вектора $M_{3}$ по алгоритму Брюно.

\begin{tabular}{|c|c|c|c|c|c|c|c|c|c|}
\hline$k$ & $M_{k}$ & & $Q_{k}$ & & & $N_{k}$ & & $\widetilde{g}_{4}^{*}$ & $M_{k} /\left(M_{k}\right)_{1}$ \\
\hline 0 & $.5746230 \mathrm{E}+1$ & 0 & 0 & 1 & 0 & 0 & 1 & -5 & 1. \\
& $.1512616 \mathrm{E}+1$ & 0 & 1 & 0 & 0 & 1 & 0 & 5 & .2632362 \\
& 1. & 1 & 0 & 0 & 1 & 0 & 0 & 5 & .1740272 \\
1 & 1. & 0 & 0 & 1 & 1 & 0 & 0 & 5 & 1. \\
& .5126157 & 0 & 1 & -1 & 1 & 1 & 0 & 5 & .5126157 \\
& .2083824 & 1 & -3 & -1 & 4 & 3 & 1 & -5 & .2083824 \\
2 & .2083824 & 0 & 0 & 1 & 4 & 1 & 1 & -5 & 1. \\
& $.9585088 \mathrm{E}-1$ & 0 & 1 & -2 & 6 & 2 & 1 & -5 & .4599759 \\
& $.7061946 \mathrm{E}-1$ & 1 & -1 & -2 & 23 & 7 & 4 & 5 & .3388936 \\
3 & $.7061946 \mathrm{E}-1$ & 0 & 0 & 1 & 10 & 9 & 4 & 5 & 1. \\
& $.2523142 \mathrm{E}-1$ & 0 & 1 & -1 & 15 & 14 & 6 & 33 & .3572871 \\
& $.1668066 \mathrm{E}-1$ & 1 & -2 & 0 & 57 & 53 & 23 & -5 & .2362048 \\
4 & $.1668066 \mathrm{E}-1$ & 0 & 0 & 1 & 43 & 29 & 10 & -5 & 1. \\
& $.8550766 \mathrm{E}-2$ & 0 & 1 & -1 & 65 & 44 & 15 & -5 & .5126157 \\
& $.3475955 \mathrm{E}-2$ & 1 & -2 & -1 & 247 & 167 & 57 & 5 & .2083824 \\
\hline
\end{tabular}

$$
T_{2}=\left(\begin{array}{ccc}
4 & -1 & 10 \\
6 & -1 & 15 \\
23 & -4 & 57
\end{array}\right)
$$

\section{СПИСОК ЦИТИРОВАННОЙ ЛИТЕРАТУРЫ}

[1] Хинчин А. Я. Цепные дроби. 3-е изд. М.: Физматгиз, 1961.

[2] Klein F. Ueber eine geometrische Auffassung der gewöhnlichen Kettenbruchentwicklung // Nachr. Ges. Wiss. Gottingen, Mathem. -Phys. Kl. 1895. № 3. P. 357-359.

[3] Klein F. Sur une representation geometrique du developpement en fraction continue ordinaire // Nouv. Ann. Math. (3). 1896. V. 15. P. 321-331.

[4] Klein F. Ausgewahlte Kapitel der Zahlentheorie. Bd. I. Einleitung. Vorlesung, 1895/1896 herausgegeben von A. Sommerfeld. Göttingen, 1896.

[5] Koksma J. F. Diophantische Approximationen. Berlin: Springer, 1936.

[6] Davenport H. On the product of three homogeneous linear forms. I // Proc. London Math. Soc. (2). 1938. V. 44. P. 412-431.

[7] Davenport H. Note on the product of three homogeneous linear forms // J. London Math. Soc. 1941. V. 16. P. 98-101.

[8] Davenport H. On the product of three homogeneous linear forms. IV // Proc. Cambrige Philos. Soc. 1943. V. 39. P. 1-21.

[9] Swinnerton-Dyer H.P.F. On the product of three homogeneous linear forms // Acta Arithmetica. 1971. V. 18. P. 371-385.

[10] Брюно А. Д., Парусников В.И. Многогранники Клейна для двух кубических форм Давенпорта // Матем. заметки. 1994. Т. 56. № 4. С. 9-27.

[11] Брюно А. Д., Парусников В.И. Сравнение разньх обобщений цепных дробей // Матем. заметки. 1997. Т. 61. № 3. С. 339-348.

[12] Брюно А. Д., Парусников В.И. Сравнение разных обобщений цепных дробей. Препринт ИПМ им. М. В. Келдыша РАН № 52, 1994.

[13] Парусников В.И. Многогранники Клейна для третьей экстремальнй кубической формы. Препринт ИПМ им. М.В. Келдыша РАН № 137, 1996. 
[14] Parusnikov V. I. Klein polyhedra for complete decomposable forms // Number Theory. Dyophantine, Computational and Algebraic Aspects / ed. K. Győry, A. Pethő, V. T. Sós. Berlin-New York: De Gruyter, 1998. P. 453-463.

[15] Парусников В.И. Многогранники Клейна с большими гранями. Препринт ИПМ им. М. В. Келдьшша РАН № 93, 1997.

[16] Парусников В.И. Многогранники Клейна для четвертой экстремальной кубической формы. Препринт ИПМ им. М. В. Келдьша РАН № 36, 1998.

[17] Голдман А., Таккер А. Многогранные выпуклые конусы // Линейные неравенства. М.: ИЛ, 1959. С. 142-161.

[18] Брюно А. Д. Локальный метод нелинейного анализа дифференциальных уравнений. М.: Наука, 1979.

[19] Гекке Э. Лекции по теории алгебраических чисел. М.-Л.: ГИТТЛ, 1940.

[20] Боревич З. И., Шафаревич И. Р. Теория чисел. 2-е изд. М.: Наука, 1972.

Институт прикладной математики им. М.В. Келдьша РАН, г. Москва Поступило E-mail : parus@applmat.msk.su 